\title{
Optical coherence tomography analysis of filtering blebs after long-term, functioning trabeculectomy and XEN ${ }^{\circledR}$ stent implant
}

\author{
Miguel A. Teus ${ }^{1,2} \cdot$ Javier Paz Moreno-Arrones ${ }^{1} \cdot$ Beatriz Castaño $^{1}$ (I) $\cdot$ Miguel A. Castejon ${ }^{1} \cdot$ Gema Bolivar $^{1}$
}

Received: 26 October 2018 / Revised: 21 January 2019 / Accepted: 11 February 2019 / Published online: 20 February 2019

(C) The Author(s) 2019

\begin{abstract}
Purpose The purpose of this study was to use Triton ${ }^{\circledR}$ SweptSource OCT to evaluate the morphology of blebs formed when eyes are treated with XEN® implants and to compare these with the blebs in successfully functioning eyes after trabeculectomy (TB) and with eyes of healthy controls.

Methods A cross-sectional, observational study. We analyzed 25 eyes, 15 after TB and 10 with XEN® implants, comparing them with 23 healthy eyes (controls). We evaluated the conjunctival morphology of the eyes using AS-OCT.

The main parameters evaluated were bleb height, sub-epithelial fibrosis, epithelial thickness, and changes in intraocular pressure (IOP).

Results We found that the filtering blebs formed in eyes in which a XEN® stent was implanted were significantly flatter (bleb height $417 \pm 183 \mu \mathrm{m}$ ) than the blebs formed in TB eyes (bleb height $618 \pm 256 \mu \mathrm{m}, p<0.05$ ). Moreover, sub-epithelial fibrosis did not develop in any of the blebs produced by the XEN stent, whereas some fibrosis was evident in $40 \%$ of the blebs that formed after TB $(p<0.05)$. The epithelium was thicker when the XEN implant was used $(65 \pm 18.5 \mu \mathrm{m})$ than when eyes underwent TB $(60 \pm 17.7 \mu \mathrm{m})$, and it was thicker than in control eyes $(51 \pm 9.7 \mu \mathrm{m}, p<0.05)$. Moreover, the decrease in the IOP induced by the $\mathrm{XEN} ®$ stent $(-8.5 \pm 5.3 \mathrm{mmHg})$ was similar to that produced by TB $(-8.8 \pm 5.2 \mathrm{mmHg}, p>0.05)$.

Conclusions Filtering blebs obtained after the introduction of a XEN® stent were morphologically distinct to those produced by $\mathrm{TB}$, and they are more similar to the healthy conjunctiva.
\end{abstract}

Keywords Bleb characteristics $\cdot$ XEN implant $\cdot$ Trabeculectomy $\cdot$ Anterior segment OCT $\cdot$ Intraocular pressure

\section{Introduction}

Glaucoma is an important cause of irreversible blindness and a major public health problem worldwide, with an increasing number of people affected by the disease [1]. Trabeculectomy (TB) is a quite effective therapy to combat glaucoma, and it is the surgical procedure most frequently performed in glaucoma patients, representing the current gold standard for surgical treatment of glaucoma [2]. However, TB requires a close post-operative follow-up due to possible

Beatriz Castaño

bea_castano90@hotmail.com

1 Department of Ophthalmology, Hospital Universitario Príncipe de Asturias, Carretera Alcalá-Meco s/n, Alcalá de Henares, 28805 Madrid, Spain

2 School of Medicine, University of Alcalá, Alcalá de Henares, Madrid, Spain complications like overfiltration and hypotony. More recently, minimally invasive glaucoma surgery (MIGS) procedures have been developed that represent safer and less aggressive alternatives to TB [3, 4], such as that involving the implantation of a XEN Gel Stent ${ }^{\circledR}$.

The success of filtering surgeries is determined by the formation of an adequate filtering bleb [5], and post-surgical evaluation of this bleb is essential to predict functionality. There are currently several ways to classify filtering blebs based on their clinical appearance when examined with a slit lamp, including the Indiana Bleb Appearance Grading Scale (IBAGS) [6] and the Moorfield's Bleb Grading System (MBGS) [7]. However, such macroscopic evaluation of the bleb alone does not allow the full extent of the changes to the conjunctiva in the filtering area to be assessed [5]. Through several studies, it has become evident that anterior segment optical coherence tomography (AS-OCT) is useful to evaluate the internal morphology of the conjunctiva and to obtain an objective measure of the size of the filtering blebs. 
Using AS-OCT, the height of the bleb, the bleb wall thickness, the reflectivity of the tissue, and the presence of epithelial microcysts can be assessed, all parameters related to the hypotensive efficacy of filtering surgeries [8-15].

The mechanism of action of the XEN Gel Stent ${ }^{\circledR}$ has been attributed to the creation of a fistula between the anterior chamber and the subconjunctival space, and since the implant is injected using an "ab interno" approach, there is no conjunctival dissection. In addition, and due to the relationship between the internal lumen diameter and the length of the implant, there is some resistance to the aqueous flow through the implant ("flow restriction" device). This phenomenon might imply that the filtering bleb that forms over the XEN implant opening may have different characteristics to those produced by conventional surgery. As such, we decided to use a DRI OCT Triton ${ }^{\circledR}$ apparatus to analyze the structural characteristics of the conjunctiva associated with filtering blebs by AS-OCT in eyes that were subjected to TB or that received a XEN Gel Stent ${ }^{\circledR}$, also comparing these to the normal conjunctiva of healthy volunteers. The data obtained highlight some of the differences in the filtering bleb that forms as a consequence of the two types of surgery, which might produce benefits in certain situations.

\section{Methods}

\section{Patients}

This is a cross-sectional, observational study that was carried out on 15 eyes with filtering blebs that formed after successful TB in which signs of filtration were evident in the slit lamp exam. Similarly, we examined 10 eyes that had been successfully implanted with a working XEN Gel Stent ${ }^{\circledR}$, and 23 eyes from healthy volunteers in which the slit lamp examination detected a normal conjunctiva. All the patients that underwent filtering surgery had been diagnosed with primary open-angle glaucoma (POAG) at the Glaucoma Unit of the Hospital Universitario Príncipe de Asturias in Alcalá de Henares (Madrid), and the surgical procedure had been performed there at least 1 year before entering the study.

We collected several clinical parameters from all participants: age, gender, the date of surgery, the type of surgery (combined surgery or stand-alone surgery), and the delay between the date of the surgery and the AS-OCT examination. Best corrected visual acuity (BCVA) was assessed using the Snellen chart, and intraocular pressure (IOP) was measured using a calibrated Goldmann applanation tonometer. The number of antiglaucoma drugs administered at the time of examination was also registered.

Patients with neck problems, those who collaborated poorly or who had difficulty in maintaining their eye in the appropriate position (gazing down), were excluded from the study.
Similarly, patients with prior ocular surgery other than the filtering procedure that might affect the conjunctiva were also excluded. When both eyes of the same subject fulfilled the inclusion criteria, only one eye (the right eye) was included.

The study adhered to the tenets of the Declaration of Helsinki and approval was obtained for the study protocol from the Ethics Committee at our institution prior to commencing enrolment. All the patients gave their informed consent after the study protocol and their rights were fully explained to them.

\section{Surgical procedures}

All TBs were performed using a fornix-based conjunctival flap and a triangular shape scleral flap that was approximately 4-mm long. Mitomycin C (MMC 0.02\%) was applied for 2 min to all the study eyes using sponges. The trabecular specimen was dissected using a sharp steel knife and a peripheral iridectomy was performed. Finally, the scleral and the conjunctival flaps were sutured using 10-0 nylon. To implant the XEN Gel Stent ${ }^{\circledR}$, a subconjunctival sclerotomy was performed, injecting the XEN 45 device into the superonasal quadrant using an "ab interno" approach. Approximately $15 \mathrm{~min}$ before inserting the implant, $0.1 \mathrm{ml}$ of a MMC solution $(0.02 \%)$ was injected into the superior conjunctiva. Both stand-alone and combined phaco-glaucoma procedures were used. In both groups of patients, the post-operative care included topic application of prophylactic antibiotics in the first week after surgery and decreasing doses of topical corticosteroids over 6 weeks.

\section{AS-OCT}

AS-OCT images of the filtering blebs were obtained using the commercially available DRI OCT Triton ${ }^{\circledR}$ (Topcon, Madrid, Spain) and based on Swept-Source OCT. According to the manufacturer, the OCT device provides high-resolution scans at a speed of 100,000 A per second when a 1050 -nm wavelength laser is used. The filtering bleb was scanned using the anterior chamber adapter provided by the manufacturer. To acquire images, the patient was asked to look down while the upper eyelid was gently pulled up to expose the filtering bleb and the surrounding superior bulbar conjunctiva, avoiding any direct pressure over the bleb area. The location raster of the AS-OCT was centered on the triangular scleral flap (Fig. 1) in the case of trabeculectomy blebs, and in the middle part of the subconjunctival implant in XEN eyes. In both cases, a radial scan of $6 \mathrm{~mm}$ perpendicular to the limbus and a second 6-mm scan tangential to the limbus centered in the area of maximal elevation of the bleb were taken (Fig. 2), as described by others [10]. At least two scans were obtained by the same operator and the best quality image was analyzed. 


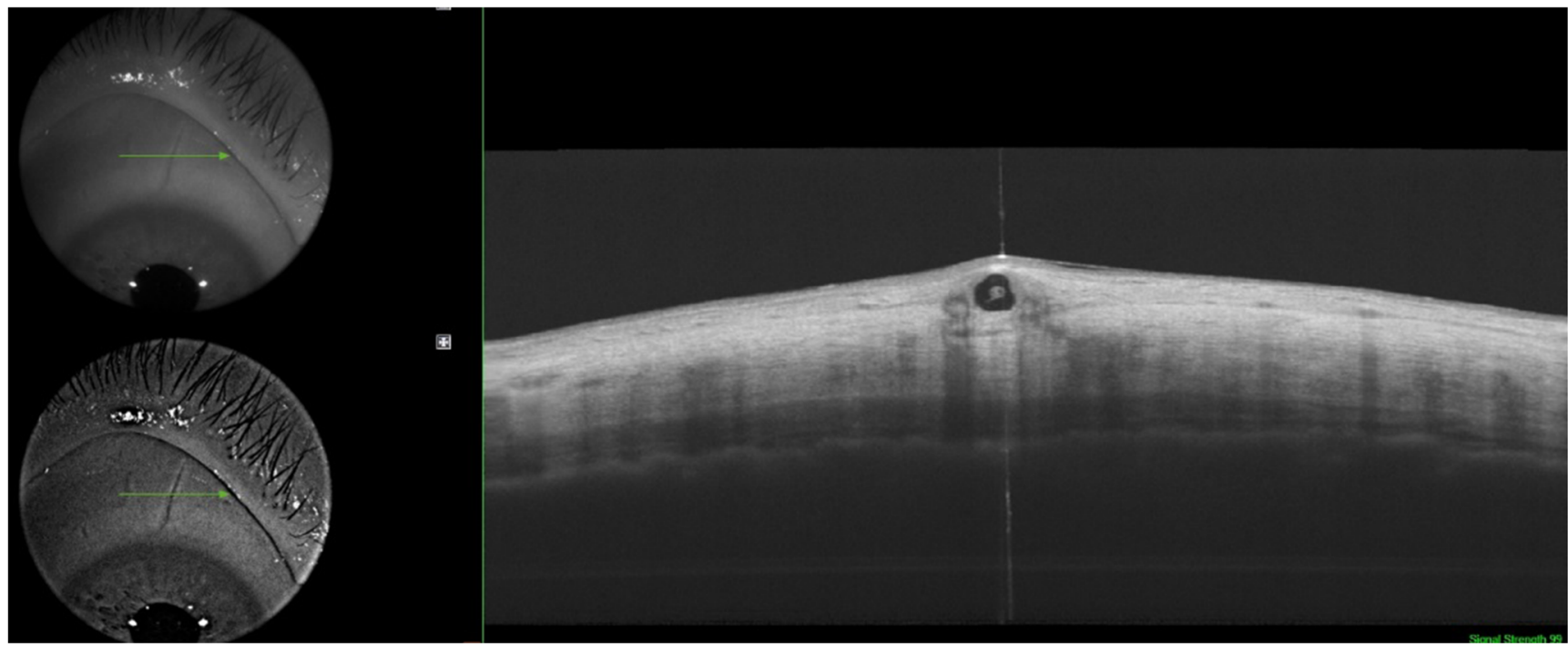

Fig. 1 Example of anterior segment OCT scan centering. The scan was centered on the scleral flap, approximately in the middle third of the filtering bleb area and in the maximum elevation (left). The right image shows the typical filtering bleb seen with the XEN Gel stent

Blebs were measured manually by two observers using the "caliper" tool of the OCT software. The researchers had no clinical information regarding the patients when they made the measurements, and in the case of any discrepancy in the measurements, both observers reanalyzed the case until a consensus was reached. The following morphological parameters of the blebs were obtained: (i) height of the bleb, defined as the maximum distance between the conjunctival surface and the underlying sclera (conjunctival thickness); (ii) thickness of the conjunctival epithelium alone (Fig. 3); (iii) the existence of hypo-reflective spaces within the conjunctiva (cysts); and (iv) areas of hyperreflectivity within the bleb (fibrosis).

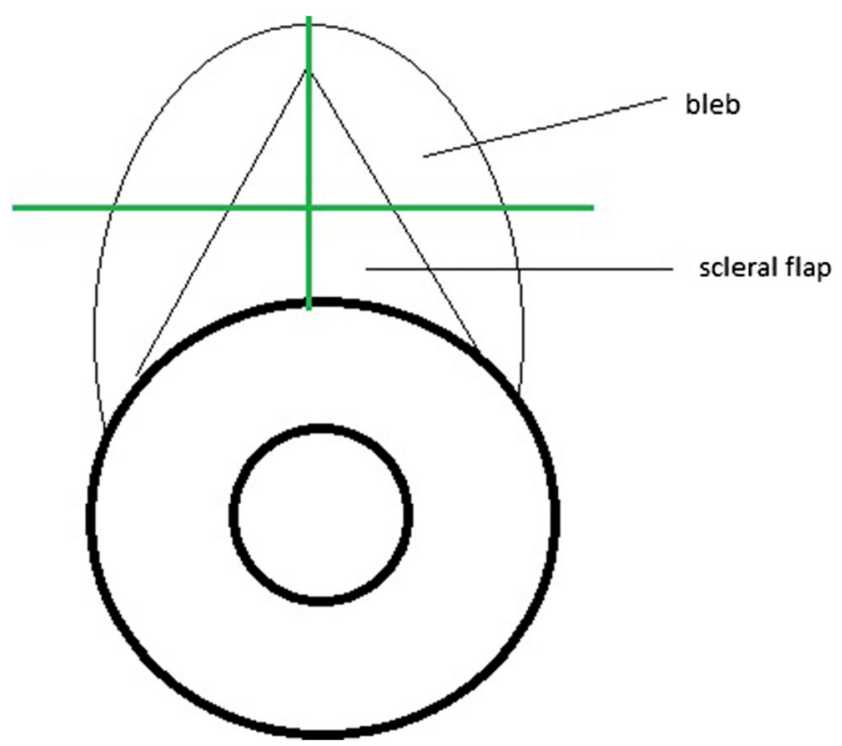

Fig. 2 Representative drawing of OCT imaging of the filtering bleb, showing the radial scan perpendicular to the limbus and the scan tangential to the limbus in the area of maximum elevation of the bleb
Additionally, the bleb morphology was carefully analyzed using the slit lamp, by the same examiner in all cases, and was described according to the Indiana Bleb Appearance Grading Scale (IBAGS) [6].

\section{Statistical analysis}

The statistical analysis was performed using Statview SE + Graphics software (Abacus concept, Cupertino CA, USA). The Mann-Whitney $U$, the Kruskal-Wallis, and the chisquare tests were used for comparisons, as appropriate, and a $p$ value less than 0.05 was considered significant.

\section{Results}

In this study, we evaluated the filtering blebs of 25 glaucoma patients that underwent surgery for glaucoma, either TB (15 eyes) or implantation of a XEN Gel Stent ${ }^{\circledR}$ (10 eyes). Moreover, the OCT data collected was compared with that obtained from the eyes of 23 healthy volunteers using the same protocol. The demographic and clinical characteristics of all the subjects were recorded in Table 1.

While there were no significant differences regarding gender and laterality (right vs. left eye) between the patient groups and the control subjects, the average age of the control group was significantly lower than that of the patients. Similarly, while the BCVA was not significantly different in the two patient groups, it was significantly higher in the control subjects relative to the glaucoma patients together, reflecting the therapeutic needs of the latter. The age differences between the control and the patient groups are unlikely to be important given that glaucoma becomes more prevalent with age. 


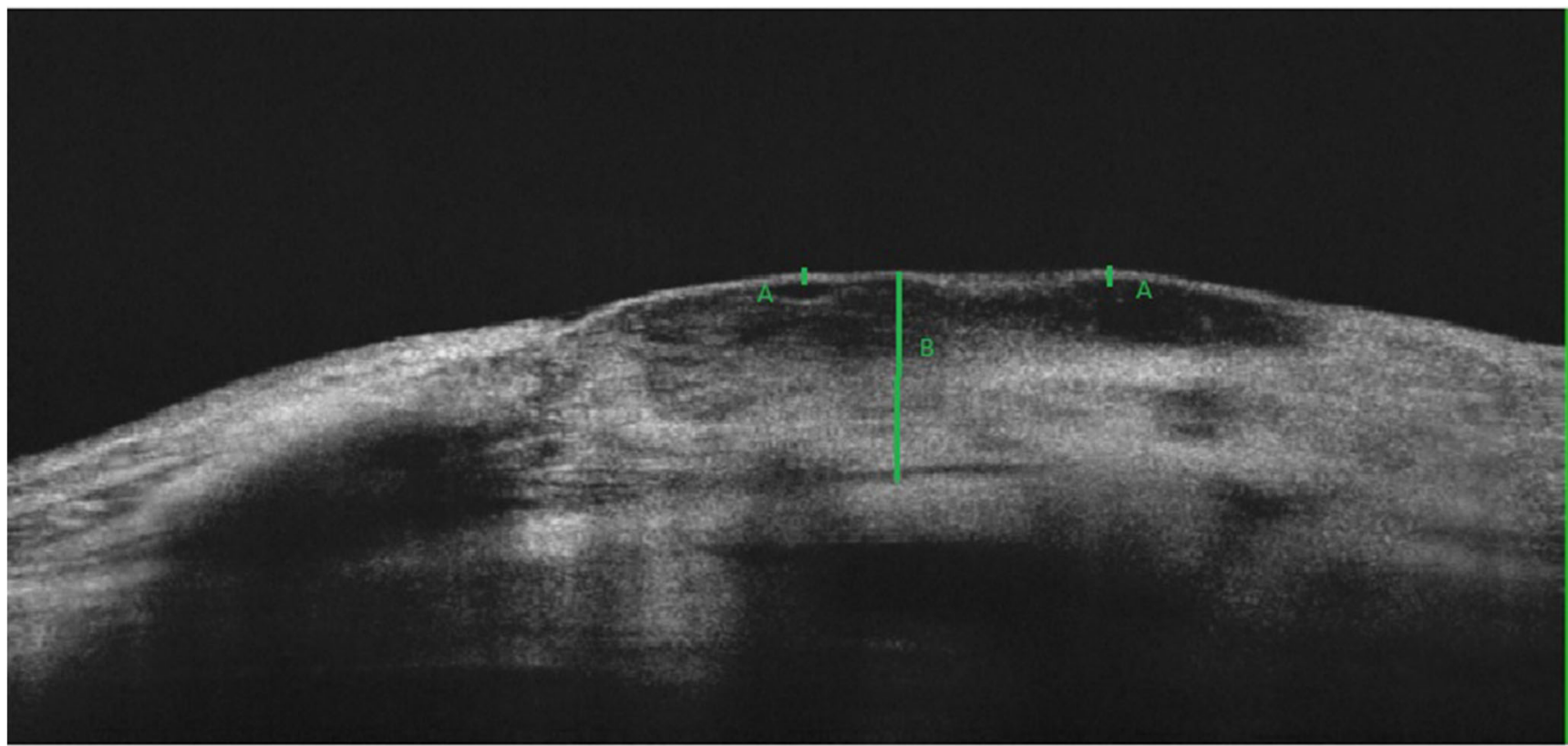

Fig. 3 Quantitative parameters of the filtration blebs. Epithelial thickness (a) and the height of the bleb (b), measured manually with the OCT caliper tool and calculated automatically with the device's software

Similarly, the higher BCVA in the control subjects would be expected given that they do not suffer from any known ocular pathology. With regards the two different groups of patients, there were no differences in terms of the other basal clinical characteristics analyzed, such as the intraocular pressure (IOP), the number of hypotensive eye drugs used, or the number of stand-alone or combined procedures.

Comparison of bleb characteristics using the IBAGS is recorded in Table 2.

The filtration blebs that formed in the 25 patients were analyzed by the same AS-OCT protocol, 15 of them appearing after TB and 10 in eyes that received a XEN Gel Stent ${ }^{\circledR}$, and they were compared with 23 eyes of healthy volunteers. There was no difference in the IOP of the glaucoma patients prior to surgery (TB $19.5 \pm 6.4 \mathrm{mmHg}$ and XEN Gel Stent ${ }^{\circledR} 19 \pm$ $4.6 \mathrm{mmHg}$ ), and both interventions produced a similar decrease in IOP (TB $-8.5 \pm 5.3$ and XEN Gel Stent ${ }^{\circledR}-8.8 \pm$
$5.2 \mathrm{mmHg}, p>0.05$ ). However, we found that when the total elevation of the conjunctiva was considered, the filtering blebs of patients that received the XEN implant were significantly flatter $(417 \pm 183 \mu \mathrm{m})$ than those that developed after TB $(618 \pm 256 \mu \mathrm{m})$, although in both cases, the conjunctiva was significantly thicker than in the controls $(244 \pm 45 \mu \mathrm{m})$. By contrast, the epithelium was significantly thicker following implantation of the XEN Gel Stent ${ }^{\circledR}(65 \pm 18.5 \mu \mathrm{m})$ than in the TB $(60 \pm 17.7 \mu \mathrm{m})$ and control subjects $(51 \pm 9.7 \mu \mathrm{m}$, $p<0.05)$.

A particularly notable feature of the filtering blebs was the complete absence of significant sub-epithelial fibrosis when the XEN stent was used, in contrast to the sub-epithelial fibrosis associated with the blebs that developed in two-thirds of the eyes subjected to TB $(p<0.05)$. Likewise, in only $50 \%$ of the eyes into which a XEN stent was introduced did "cystic areas" develop, as opposed to their development in $80 \%$ of the
Table 1 Main clinical parameters and demography of the patients. $B C V A$, best corrected visual acuity; $M$, male; $F$, female; $O D$, oculus dexter; $O S$, oculus sinister; $I O P$, intraocular pressure; IOP dif, IOP decrease induced by surgery; Phaco, phacoemulsification surgery; $T B$, trabeculectomy

\begin{tabular}{lllll}
\hline & TB & XEN & Control & $p$ value \\
\hline Number of eyes & 15 & 10 & 23 & \\
Age (years) & $70.19 \pm 9.61$ & $72.7 \pm 12.51$ & $62.3 \pm 10.6$ & 0.01 \\
BCVA & $0.72 \pm 0.23$ & $0.73 \pm 0.14$ & $0.88 \pm 0.09$ & 0.01 \\
Sex (M/F) & $11 / 4$ & $7 / 3$ & $9 / 14$ & 0.1 \\
Eye (OD/OS) & $8 / 7$ & $5 / 5$ & $12 / 11$ & 0.9 \\
IOP dif (mmHg) & $-8.5 \pm 5.3$ & $-8.8 \pm 5.2$ & & 0.9 \\
IOP pre-surgery (mmHg) & $19.5 \pm 6.4$ & $19 \pm 4.6$ & & 0.8 \\
Number of eye drops & $0.33 \pm 0.6$ & $0.3 \pm 0.4$ & & 0.8 \\
Phaco (yes/no) & $12 / 3$ & $5 / 5$ & & 0.7 \\
Time since surgery (months) & $36 \pm 0.82$ & $44 \pm 20.19$ & & 0.6 \\
\hline
\end{tabular}


Table 2 Morphology and bleb characteristics using the Indiana Bleb Appearance Grading Scale (IBAGS)

\begin{tabular}{llc}
\hline & Trabeculectomy (15) & XEN (10) \\
\hline Bleb height & & \\
H0: flat bleb & $2(13.3 \%)$ & $4(40 \%)$ \\
H1: low bleb & $4(26.67 \%)$ & $6(60 \%)$ \\
H2: medium bleb & $5(33.3 \%)$ & $0(0 \%)$ \\
H3: high bleb & $4(26.67 \%)$ & $0(0 \%)$ \\
Horizontal extent & & \\
E0: $0<1$ clock hours & $0(0 \%)$ & $0(0 \%)$ \\
E1: $1-2$ clock hours & $0(0 \%)$ & $2(20 \%)$ \\
E2: $>2<4$ clock hours & $6(40 \%)$ & $5(50 \%)$ \\
E3: 4 or > clock hours & $9(60 \%)$ & $3(30 \%)$ \\
Bleb vascularity & & $0(0 \%)$ \\
VO: avascular white & $4(26.67 \%)$ & $2(20 \%)$ \\
V1: avascular cystic & $3(20 \%)$ & $5(50 \%)$ \\
V2: mild vascularity & $5(33.3 \%)$ & $3(30 \%)$ \\
V3: moderate vascularity & $3(20 \%)$ & $0(0 \%)$ \\
V4: extensive vascularity & $0(0 \%)$ & $0(0 \%)$ \\
Seidel test & & $0(0 \%)$ \\
S0: no leak & $15(100 \%)$ & \\
S1: multiple pinpoint leaks & $0(0 \%)$ & \\
S2: streaming leak (within 5 s) & $0(0 \%)$ & \\
\hline
\end{tabular}

eyes subjected to TB $(p<0.05)$. Moreover, these hyporeflective areas were typically much smaller in the eyes that received the XEN stent than the larger cystic cavities evident after TB, as it is shown in Table 3.

\section{Discussion}

In this study, we demonstrate the specific characteristics of the blebs produced in a filtering conjunctiva following implantation of a XEN Gel Stent ${ }^{\circledR}$ to control IOP, comparing the effects of this approach with those of TB and with the characteristics of control untreated eyes. In this sense, we show that the filtering blebs that form when using the XEN Gel Stent ${ }^{\circledR}$ are smaller and with fewer intrableb cystic cavities, and that they are associated with less fibrosis and a thicker epithelium than filtering TBs. Nevertheless, the conjunctiva over the XEN implant is clearly distinct to that of a normal, healthy conjunctiva, indicating that the XEN implant induces the development of a "filtering conjunctiva" and not a conventional, localized bleb.

In recent years, the XEN Gel Stent ${ }^{\circledR}$ and other MIGS devices have appeared as alternatives to clinically lower IOP in a safer way than TB when treating glaucoma. One advantage of the XEN Gel Stent ${ }^{\circledR}$ is that it is used "ab interno," which implies less surgical trauma and fewer time-consuming procedures, such that it has lower rates of intra- and postoperative complications $[3,4]$. Thus, the use of this device is associated with a less aggressive manipulation of the conjunctiva, which may be relevant in terms of avoiding subconjunctival bleeding and conjunctival perforation [4]. In addition, the preservation of the conjunctival lymphatic vessels may facilitate more physiological absorption of the aqueous humor [16].

Conjunctival fibrosis has been associated with failure of filtration surgery and it is defined as the presence of areas with high reflectivity within the bleb, probably due to the high density of the connective tissue [9-15]. In the current study, we found areas of high reflectivity in $40 \%$ of TBs but not in the blebs that developed after implantation of the XEN Gel Stent ${ }^{\circledR}$. An earlier study of the blebs formed following XEN Gel Stent ${ }^{\circledR}$ implantation highlighted that in the majority of cases, their filtration characteristics suggested they functioned well [17], as found in all the XEN blebs studied here. However, only the internal reflectivity of the bleb was evaluated in this previous study, and quantitative parameters thought to be predictors of good aqueous humor drainage were not analyzed [8-15].

The evaluation of blebs has traditionally been based on subjective evaluation by the physician at the slit lamp. As such, the classification systems currently used assess the height, extension, and vascularization [6,7]. Our results using the IBAGS classification indicate that the blebs in trabeculectomy eyes were higher, broader, and with less vascularity than XEN eyes. It is clear that AS-OCT offers several advantages over slit lamp examination to accurately measure the bleb size or to evaluate the internal structures of the filtration blebs [9-15]. The filtering blebs obtained with the XEN Gel Stent ${ }^{\circledR}$ have previously been studied by AS-OCT [18], indicating that non-functioning blebs were flatter and with a higher bleb wall reflectivity. Well-functioning XEN blebs also had a low degree of reflectivity, although there was no control group in this earlier study, and no comparison with other filtering procedures or with a healthy conjunctiva was possible.
Table 3 Morphology of the filtering blebs (all values in microns)

\begin{tabular}{lllll}
\hline & TB & XEN & Control & $p$ value \\
\hline Bleb height (conjunctival thickness) & $618 \pm 256$ & $417 \pm 183$ & $244 \pm 45$ & 0.01 \\
Epithelial thickness & $60 \pm 17.7$ & $65 \pm 18.5$ & $51 \pm 9.7$ & 0.04 \\
Fibrosis (yes/no) & $6 / 9$ & $0 / 10$ & $0 / 23$ & 0.005 \\
Cysts (yes/no) & $12 / 3$ & $5 / 5$ & $0 / 23$ & 0.04 \\
\hline
\end{tabular}


It is also interesting that functioning blebs have numerous intrableb "cystic" spaces or areas of hypo-reflectivity in ASOCT scans, as seen previously [13-15]. These areas seem to correspond to the aqueous humor that accumulates at this intra-conjunctival level, and we found that the XEN blebs had "microcystic" intrableb areas that were smaller than the larger cystic cavities seen in the TB blebs.

Functioning blebs also appear to have thicker walls than non-functioning ones $[9,10,15]$ and we also found thicker walls in the blebs formed with the XEN Gel stent, possibly reflecting the presence of intraepithelial microcysts that increase the epithelial thickness. The distinct behavior of the conjunctiva could be explained by the flow resistance associated with the XEN implant, a function of the length and small internal lumen diameter that is reflected by the HagenPoiseuille equation [19]. Thus, it is possible that the relative restriction to flow of the XEN stent limits the liquid pressure in the subconjunctival space, and that this gives the conjunctiva a more physiological appearance in these eyes. In addition, since no dissection is performed and no cauterization is used, the conjunctiva and its vessels are preserved during XEN Gel stent ${ }^{\circledR}$ surgery. Thus, the aqueous humor can drain through conjunctival diffusion or by entering the untouched conjunctival lymphatic vessels [16]. Therefore, we consider that a normal looking diffuse "filtering" conjunctiva, without a localized "bleb," can be obtained with good surgical control of the IOP. Furthermore, a flatter "filtering" area would produce less ocular surface discomfort and possibly, less risk of infection than conventional filtering blebs.

This study has some limitations and not least, that it was not carried out blind to the experimental manipulations. However, since the implant is readily visible in the OCT images obtained, it is difficult to carry out such studies blind to the experimental conditions. Nevertheless, we tried to improve the accuracy of the measurements by using two independent observers who follow the same protocol. In addition, we must bear in mind any possible artifacts in the AS-OCT evaluation of the blebs, and to minimize these, we obtained the images when focusing the scan on the same reference points. By contrast, one of the strengths of the current study is that it provides comparative data of two functioning filtering surgeries: TB with evident bleb and visible liquid in the subconjunctival space (evidence of filtration); and XEN implant blebs that often have the appearance of a normal conjunctiva upon slit lamp examination. We also included a control group in the study, and although this group was not agematched to either of the experimental groups, these control subjects were younger than either of the glaucoma patient groups and as such, they are less likely to suffer from eye pathologies and glaucoma in particular.

While the data presented here suggest that the use of XEN Gel stent ${ }^{\circledR}$ surgery may offer certain advantages over TB in managing the IOP associated with glaucoma, it is clear that more research is needed in this field to expand our knowledge about the conjunctival response to surgical filtering procedures.

Acknowledgements The authors wish to thank the staff at the Hospital Universitario Príncipe de Asturias who assisted us in performing this study and the patients who agreed to participate in the study, without whom this would not have been possible. The authors also acknowledge the assistance of Allergan S.A. who financed a medical writer (Biomedred SL) to aid us in the preparation of this manuscript. This assistance did not influence the manner in which the data was presented and Allergan had no say in the design or conduct of this research, or in the conclusions reached.

Compliance with ethical standards Allergan S.A. who financed a medical writer (Biomedred SL) to aid us in the preparation of this manuscript. This assistance did not influence the manner in which the data was presented and Allergan had no say in the design or conduct of this research, or in the conclusions reached.

Conflict of interest Author Miguel A. Teus reports grants and personal fees from Johnson \& Johnson, personal fees and non-financial support from Alcon, personal fees and non-financial support from Allergan, personal fees and non-financial support from Novartis, outside the submitted work.

Authors Javier Paz Moreno-Arrones, Beatriz Castaño MD, Miguel A. Castejon, and Gema Bolivar declare that they have no conflict of interest.

Ethical approval The study protocol was approved by the Ethics Committee of our institution and all procedures performed involving human participants followed the 1964 Helsinki declaration and its later amendments or comparable ethical standards.

Informed consent was obtained from all individual participants included in the study.

Open Access This article is distributed under the terms of the Creative Commons Attribution 4.0 International License (http:// creativecommons.org/licenses/by/4.0/), which permits unrestricted use, distribution, and reproduction in any medium, provided you give appropriate credit to the original author(s) and the source, provide a link to the Creative Commons license, and indicate if changes were made.

Publisher's note Springer Nature remains neutral with regard to jurisdictional claims in published maps and institutional affiliations.

\section{References}

1. Tham YC, Li X, Wong TY, Quigley HA, Aung T, Cheng CY (2014) Global prevalence of glaucoma and projections of glaucoma burden through 2040: a systematic review and meta-analysis. Ophthalmology 121:2081-2090

2. Kirwan JF, Lockwood AJ, Macleod A, Broadway DC, King AJ et al (2013) Trabeculectomy in the 21st century: a multicenter analysis. Ophthalmology 12:2532-3539

3. Ritcher GM, Coleman AL (2016) Minimally invasive glaucoma surgery: current status and future prospects. Clin Ophthalmol 10: 189-206

4. De Gregorio A, Pedrotti E, Russo L, Morselli S (2017) Minimally invasive combined glaucoma and cataract surgery: clinical results of the smallest ab interno gel stent. Int Ophthalmol 29 
5. Pitch G, Grehn F (1998) Classification of filtering blebs in trabeculectomy: biomicroscopy and functionality. Curr Opin Ophthalmol 9:2-8

6. Cantor L, Mantravadi A, WuDunn, et al. (2003) Morphologic classification of filtering blebs after glaucoma filtration surgery: the Indiana Bleb Appearance Grading Scale. J Glaucoma; 12: 266-271

7. Wells AP, Crowston JG, Marks J et al (2004) A pilot study of a system for grading of drainage blebs after glaucoma surgery. $\mathrm{J}$ Glaucoma 13:454-460

8. Narita A, Morizane Y, Miyake T, Seguchi J, Baba T, Shiraga F (2017) Characteristics of successful filtering blebs at 1 year after trabeculectomy using swept-source three-dimensional anterior segment optical coherence tomography. Jpn J Ophthalmol 61(3):253259

9. Hamanaka T, Omata T, Sekimoto S, Sugiyama T, Fujikoshi Y. (2013) Bleb analysis by using anterior segment optical coherence tomography in two different methods of trabeculectomy. Invest Ophthalmol Vis Sci 2013;54:6536-6541

10. Tominaga A, Miki A, Yamazaki Y, Matsushita K, Otori Y (2010) The assessment of the filtering bleb function with anterior segment optical coherence tomography. J Glaucoma 19:551-555

11. Napoli PE, Zucca I, Fossarello M (2014) Qualitative and quantitative analysis of filtering blebs with optical coherence tomography. Can J Ophthalmol 49:210-216

12. Kokubun T, Kunikata H, Tsuda S, Himori N, Maruyama K, Nakazawa T (2016) Quantification of the filtering bleb's structure with anterior segment optical coherence tomography. Clin Exp Ophthalmol 44:446-454

13. Meziani L, Tahiri Joutei Hassani R, El Sanharawi M, Brasnu E, Liang H, Hamard P, Baudouin C, Labbe A (2016) Evaluation of blebs after filtering surgery with en-face anterior-segment optical coherence tomography: a pilot study. J Glaucoma 25:e550-e558

14. Ciancanglini M, Carpintero P, Agnifili L, Nubile M, Lanzini M, Fasanella V, Mastropasqua L (2008) Filtering bleb functionality: a clinical, anterior segment optical coherence tomography and in vivo confocal microscopy study. J Glaucoma 17:308-317

15. Güven Y1lmaz S, Değirmenci C, Palamar M, Yağc1 A (2015) Evaluation of filtering bleb function after trabeculectomy with Mitomycin C using biomicroscopy, anterior segment optical coherence tomography and in vivo confocal microscopy. Turk $\mathrm{J}$ Ophthalmol 45:132-137

16. Yu DY, Morgan WH, Sun X, Su EN, Cringle SJ, Yu PK, House P, Guo W, Yu X (2009) The critical role of the conjunctiva in glaucoma filtration surgery. Prog Retin Eye Res 28:303-328

17. Olate-Pérez Á, Pérez-Torregrosa VT, Gargallo-Benedicto A, NeiraIbáñez P, Cerdà-Ibáñez M, Osorio-Alayo V, Barreiro-Rego A, Duch-Samper A (2017) Prospective study of filtering blebs after XEN45 surgery. Arch Soc Esp Oftalmol 92:366-371

18. Fea AM, Spinetta R, Cannizzo PML, Consolandi G, Lavia C, Aragno V, Germinetti F, Rolle T (2017) Evaluation of bleb morphology and reduction in IOP and glaucoma medication following implantation of a novel gel stent. J Ophthalmol 9364910

19. D'Alessandro E, Guidotti JM, Mansouri K, Mermoud A (2017) XEN-augmented Baerveldt: a new surgical technique for refractory glaucoma. J Glaucoma 26:e90-e92 